$\stackrel{W}{=}$

Global burnals Inc.

है

\title{
Search of Ways to Improve the Efficiency of the Diagnostic Results and the Quality of Treatment of Dystrophy and Vulva Cancer
}

By Cherenkov V. G., Sannikova M. V., Alexandrova I. V., Pacewicz K. G K. G. Pacwich \& Egorova E. S.

Jaroslav-the- Wise Novgorod State University Abstract- With vulvar dysplasia, sclerotic deprive and suspected cancer under our observation there were respectively 115 and 97 patients. The effectiveness of treatment of vulva dystrophy (VIN II-III degree) by the method of photodynamic therapy (PDT) 45.8=4.7\%. However, dysplasia II - III degree, sclerotic changes with the formation of pronounced horn scales prevents the full PDF and recurrence of the disease. Therefore, the treatment of choice is surgical treatment with a reconstructive plastic. In order to reduce bleeding and antiblastic we used krioapplikatsiya, apparate "Harmonics", and the decay of tumor angiographic chemoembolization before surgery. The use of new reconstructive plastic surgery, including with the use of abdominal skin and fascial flap combined with vascularized lower segments of the rectus muscles (patent for invention № 2580665 from 11.11.14), have helped to reduce complications, improve cosmetic effect and reduce the duration of lymphorrhea in 2-3 days.

Keywords: vulvar cancer, PDT, cryoapplication, apparate "Harmonics", angiohemoembolization, reconstructive plastic surgery of the abdominal skin and lower segments of the rectus muscles.

GJMR-F Classification: NLMC Code: WB 141

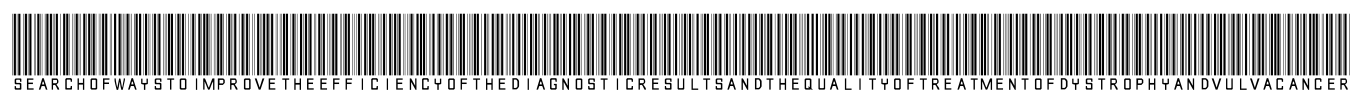

Strictly as per the compliance and regulations of:

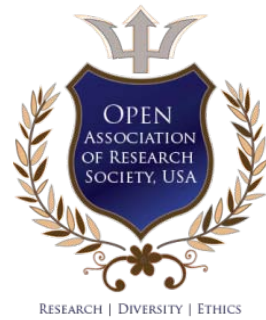

(c) 2019. Cherenkov V. G., Sannikova M. V., Alexandrova I. V., Pacewicz K. G K. G. Pacwich \& Egorova E. S. This is a research/review paper, distributed under the terms of the Creative Commons Attribution-Noncommercial 3.0 Unported License http://creativecommons.org/licenses/by-nc/3.0/), permitting all non-commercial use, distribution, and reproduction in any medium, provided the original work is properly cited. 


\title{
Search of Ways to Improve the Efficiency of the Diagnostic Results and the Quality of Treatment of Dystrophy and Vulva Cancer
}

\author{
Cherenkov V. G. ${ }^{\alpha}$, Sannikova M. V. ${ }^{\sigma}$, Alexandrova I. V. ${ }^{\rho}$, Pacewicz K. G K. G. Pacwich ${ }^{\omega}$ \& Egorova E. S. ${ }^{*}$
}

Abstract- With vulvar dysplasia, sclerotic deprive and suspected cancer under our observation there were respectively 115 and 97 patients. The effectiveness of treatment of vulva dystrophy (VIN II-III degree) by the method of photodynamic therapy (PDT) $45.8=4.7 \%$. However, dysplasia II - III degree, sclerotic changes with the formation of pronounced horn scales prevents the full PDF and recurrence of the disease. Therefore, the treatment of choice is surgical treatment with a reconstructive plastic. In order to reduce bleeding and antiblastic we used krioapplikatsiya, apparate "Harmonics", and the decay of tumor angiographic chemoembolization before surgery. The use of new reconstructive plastic surgery, including with the use of abdominal skin and fascial flap combined with vascularized lower segments of the rectus muscles (patent for invention № 2580665 from 11.11.14), have helped to reduce complications, improve cosmetic effect and reduce the duration of lymphorrhea in 2-3 days.

Keywords: vulvar cancer, PDT, cryoapplication, apparate "Harmonics", angiohemoembolization, reconstructive plastic surgery of the abdominal skin and lower segments of the rectus muscles.

\section{INTRODUCTION}

$\longrightarrow$ ancer of the external genitals is up to $8 \%$ in the overall structure of the incidence of malignant neoplasms of female genital organs, relapses that occur within the first 5 years to $60 \%$. [1]. Vulva cancer $(\mathrm{CV})$ is mainly detected in elderly menopausal women. Unfortunately, it is increasingly common at a younger age, associated with the increasing threat of papillomavirus infection [2]. And this is a reality that we have to reckon with. Features of development, multicentricity, and often diffusivity of the lesion against the background of diffuse dysplastic changes and sclerotic lichen or papillomatosis create certain difficulties in the early stages of diagnosis [8]. Given the above, we used the technique of scraping with a scalpel consisting of two stages: 1) removal (scraping) Horny scales of the epithelium of the 4-5 most suspicious areas; 2) scarification and obtaining cells from deep layers within the basal layer before the appearance of "dews" blood. Cytological examination of vulvar dysplasia allowed at $76.0 \pm 3.4 \%$ of cases to establish a correct diagnosis, including 13 cases against this background, the identified cancer (0-1 stages, which is significantly higher than the cumulative literature data (up to 57\%) [3]. The study of methods of reconstructive plastic surgery (RPO) with a skin-fascial flap from the posterior thigh was started in the 80s years last century by Knapstein P. G [9].

The possibilities of treatment of vulvar dysplasia and cancer are contradictory.

\section{Aim}

Assessment and the role of the VDT and the search of ways of increase of efficiency of diagnostics and surgical procedures in complex therapy of vulvar dystrophies and vulvar cancer.

\section{ili. Material and Methods}

With vulvar dysplasia and sclerotic lichen under our supervision were 115 patients aged 46 to 82 years and 9 patients in combination with carcinoma in situ and RV 1 -II stages. Mean age $55 \pm 3.6 \%$. PDT was performed in 43 patients with diffuse vulvar dysplasia, incl. (VIN I - 27, VIN II - 16) and in RV in order to prepare the surrounding tissues (in the transition of sclerotic changes) on the skin of inguinal masonry and inner thighs), incl. in 2 patients with relapse of $\mathrm{CV}$. 


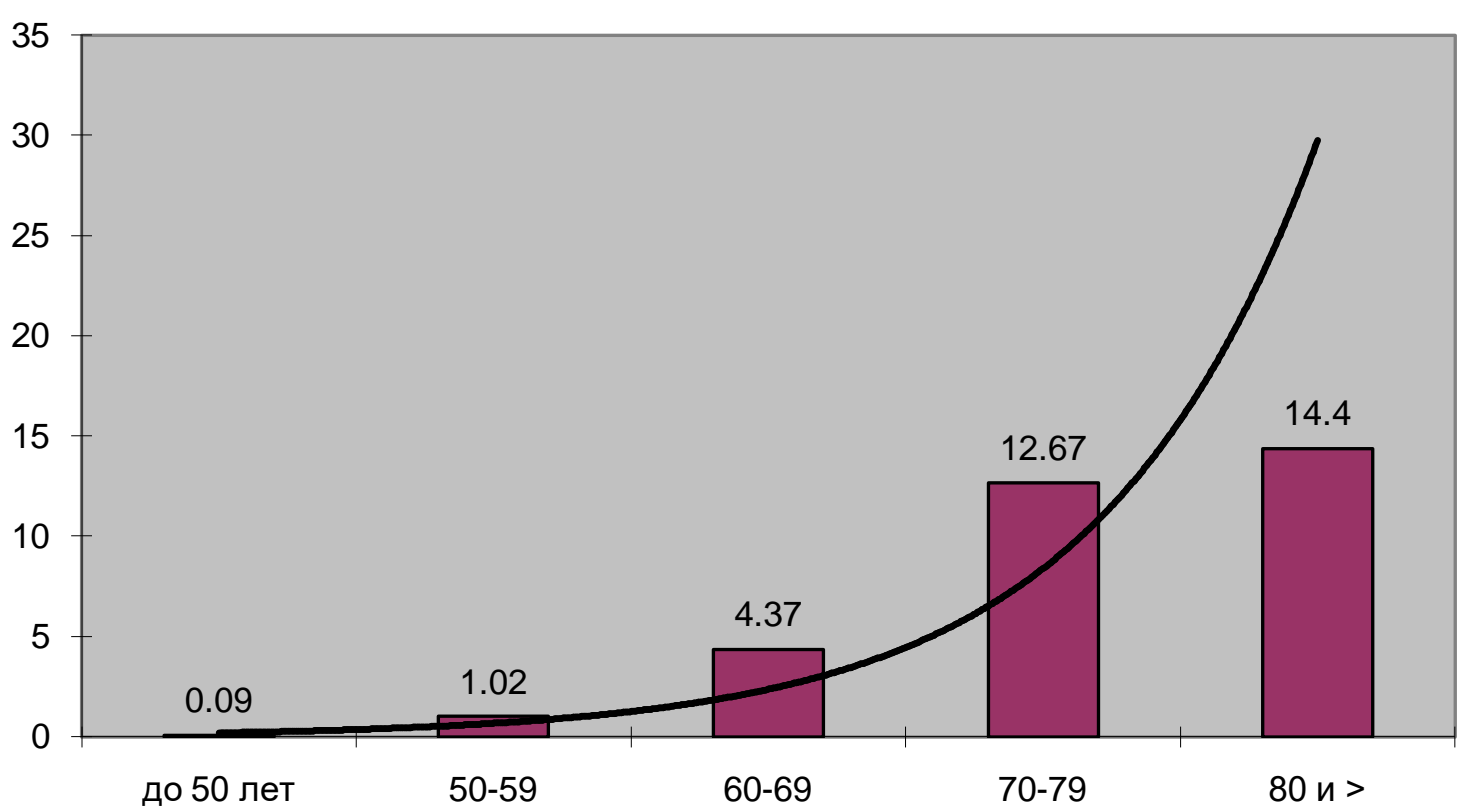

Fig. 1: The incidence of CV diseases per 100000 of the female population of the region by age groups on average for the last 5 years (2014-2018).

In our study we used a second generation sensitizer - photoditazine. PDT session was performed 1.5-2 hours after intravenous drip infusion of the drug (VETA-Grand LLC) at the rate of an average of $1.0 \mathrm{mg} / \mathrm{kg}$ body weight in $100 \mathrm{ml}$ of $0.9 \%$ sodium chloride solution in a semi-darkened room. Laser irradiation using semiconductor device "Atkus - 2" with energy density of $80-250 \mathrm{j} / \mathrm{cm}^{2}$ and exposure time from 10 to $30 \mathrm{~min}$. PDT allowed to cure VIN I in $21(77.7=2.2 \%)$ and only in 2 of $16(12.5=0.5 \%)$ women with VIN II degree.

However, after 5 years, the resumption of skin itching and residual focal leukoplakia were noted in 5 women, which is associated with insufficient exposure to laser exposure to each zone, in the presence of pronounced Horny scales. In addition, PDT by moving the laser beam by hand does not avoid subjectivity, and means of disease recurrence.

5 -year relapse-free survival was found in 16 of $27(59.2 \pm 3.6 \%)$ women with predominantly VIN I. In situations with diffuse or transient II - III degree dysplasia behind the femoral fold, in our opinion, a combined approach is required in the choice of treatment depending on the prevalence of the process (PDT+ vulvectomy with RPO).

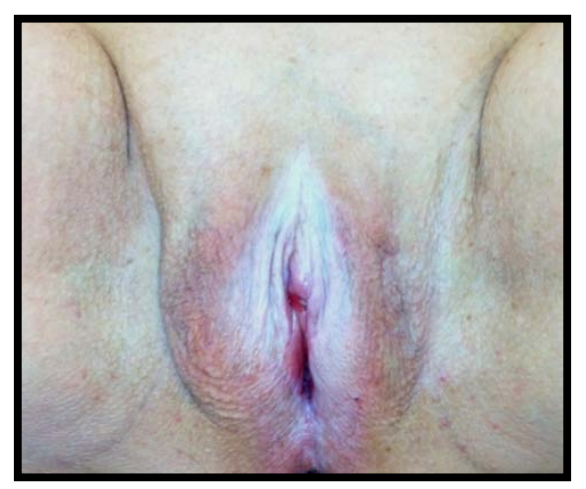

Fig. 2: Cancer in situ on the background of diffuse sclerotic lichen and vulvar dysplasia, passing to the skin of the thigh (P., 62 years).

As note themselves women after PDT was only a temporary subjective effect, after which itching resumed and they resorted to various popular their means. Plasty with full-fledged skin-fascial flaps with good vascularization from the posterior-medial surface of the thigh in 97 patients showed that it is pathogenic, since one of the main reasons for the development of VIN I-III is the sclerosis of local vessels and tissue atrophy. All patients underwent expanded vulvectomy and bilateral or unilateral inguinal-femoral lymph node dissection depending on the results of ultrasound and MRI.

Despite the obliteration of arterial vessels in the formation of tabloid dystrophy, due to anatomical features in the area of the vulva is not only preserved, but also expanded venous plexus with outflow through $\mathrm{V}$. pudendi interna. In order to avoid dispersion and devitalization of the tumor cells (ablastic and antiblastic) 
excision always conducted radiowave scalpel and apparatus "Harmonics", making the preliminary incision of the skin usual scalpel.

Recently, the excision of the tumor in 37 women was preceded by cryoapplication and credibilitate to $\mathrm{t}-185^{\circ}$ through the entire thickness of the tumor using the apparatus ERBE-6, exposure 5-10 min (Fig. 3). The data show that this approach is promising and does not affect healing. Surgical excision is almost without bloodshed on the part of the tumor. In the latter group, within 5 years, relapses occurred only in 3 patients $(8.1 \pm 0.9 \%)$, which is significantly lower than in the literature.

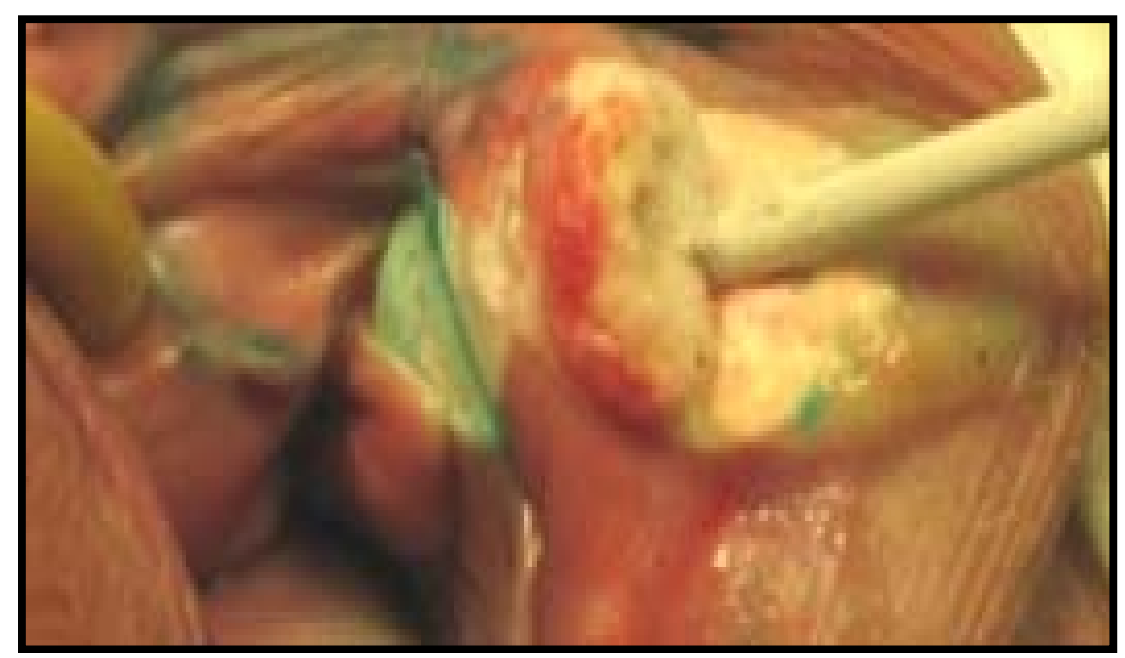

Fig. 3: Krioapplikatsiya and freezing the tumor.

In three patients with RV T3n2mo with tumor decay and bleeding, we undertook angiographic

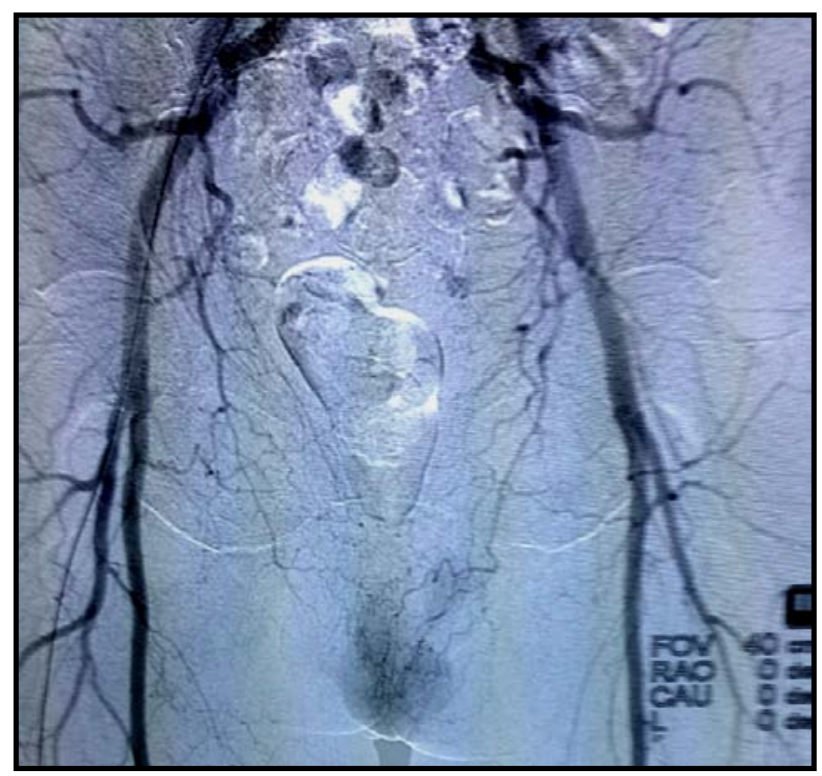

a) chemoembolization (HAE) with the introduction of 30 mg doxorubicin (Fig. 4).

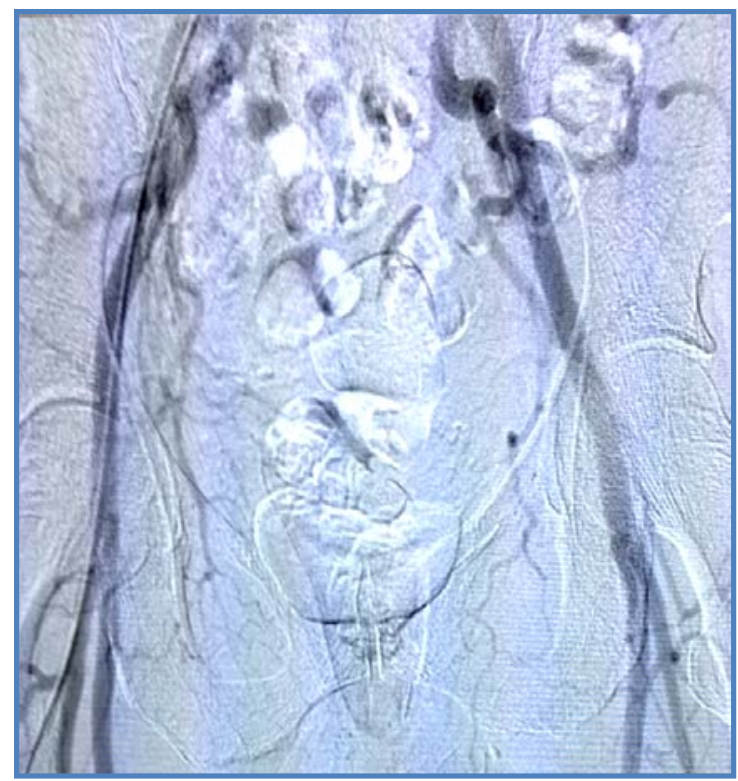

b)

Fig. 4: Angiographic chemoembolization a. pudenda interna: (a) angiography to HAE; b) angiography after HAE).

And although the diameter of a. pudenda interna did not allow to hold the catheter to the focus of pathology. Partial HAE it possible to reduce bleeding, restore hemoglobin by additional hemotransfusion and perform expanded vulvectomy and bilateral lymph node dissection. 


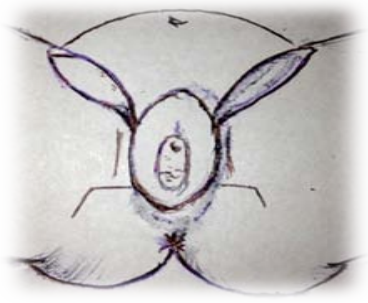

a)

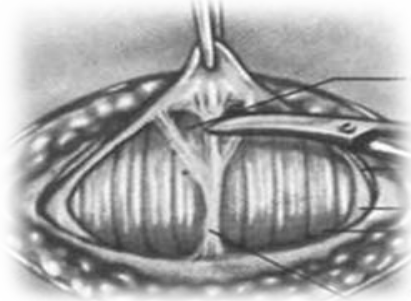

b)

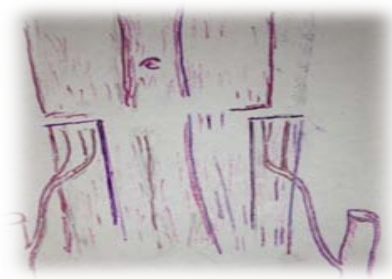

c)

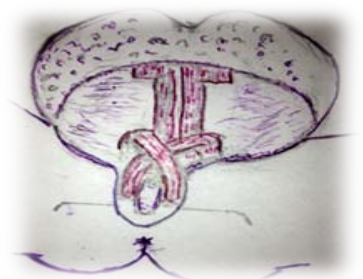

d)

Fig. 5: (a) excision of the vulva and inguinal areas; (b) and (c) Pfannenstiel incision exposing the rectus muscles and (a) epigastrica inferior; (d) formation of the labia majora by cross-segments of the rectus muscles.

With the aim of forming the volume of the external genitalia, improve the appearance quality and reduce lymphorrhea recently we have developed a method rekonstruktivnoi plastics (Patent No. 2580665 [4]) of the external genitalia through the mobilization of abdominal skin and fascial flap and the lower segments of the rectus muscles along with a.epigastrica inferior (Fig 5 a, b, c and d) using the apparatus "Harmonics". Closure of the wound was carried out at the beginning cross mobilized segments of direct muscles of a stomach on the vascular pedicle. The latter create the missing volume of the labia majora and represent a unique plastic material for vascularization and lymph drainage. In the area of the intersection of muscles and ends of the segment, fixation to the muscles of the vagina was performed by dissolving seams so that they did not hang over the mouth of the urethra and did not close the symphysis of the pubis. The next stage was sutured fascia of the rectus muscles of the abdominal wall. Then, 6-7 stitches were applied along the Donati to the skin of the perineum and the back wall of the vagina without tension in order to determine to what level the skin defect will be filled with an abdominal skin-fascial flap without tension.

Abdominal skin-fascial flap was placed on the wound surface, adapting it by cutting off excess and sharp skin areas, sutures were applied. Determining the projection of the abdominal flap, adjacent to the pubis, imposes two provisory internal anchor sutures to the periosteum, which is then stitched to the abdominal flap (without skin), genital forming a fold. Then through a separate puncture of the abdominal flap in the inguinal areas has introduced an active drainage in the inguinalfemoral area. Then every $0.8 \mathrm{~cm}$ for the tightness of stitches on the skin and the vaginal mucosa around the entire circumference and nodal skin sutures for Donati.

The operations were performed in 12 women, mostly aged $45-55$ years with abdominal obesity. The process was localized on the skin and mucous membrane of the anterior half of the vulva. In one case, focal leukoplakia was an independent disease, in another case, an initial cancer was diagnosed. Healing took place by primary tension, except for 1 woman $(8.3 \pm 1.4 \%)$ with obesity and type II diabetes mellitus. An important aspect of reconstructive vulvectomies by abdominal flap in combination with segments of straight muscles on the vascular pedicle was a decrease in the duration of lymphorhea for 2-3 days and the formation of the appearance of the organ.

\section{Discussion And Conclusions}

As a result of the work carried out, mortality from CV ( Fig. 6), despite the increase in morbidity, since 2005, when plastic surgery was introduced and modern approaches to tumor devitalization decreased by 6.2 times.

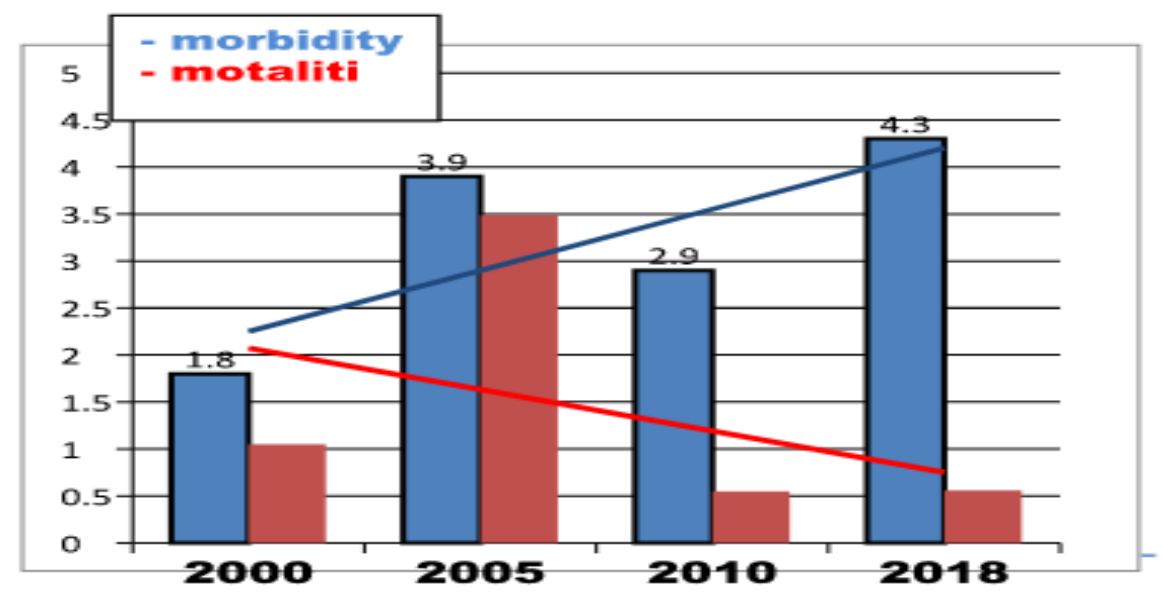

Fig. 6: Morbidity and mortality from cancer of the vulva (100 000 female population) in Novgorod region. 
Thus, further ways to improve the results of treatment lie, on the one hand, on the application and improvement of modern technologies, in particular PDT, cryodevitalization, apparatus "Harmonics" and reconstructive plastic surgery, and on the other, to improve cancer literacy of the population and General practitioners.

The use of reconstructive plastic operations in particular with the use of vascularized lower the segments of the rectus abdominis muscle is an individual method choice in young women with cancer of the vulva and vulgar dystrophy (VIN II-III), a measure of prevention of invasive forms cancer, helps to reduce complications, increase cosmetic effect and reduce the duration of lymphorrhea on average for 3-4 days.

Requires further study of the possibilities of the use of angiohemoembolization in neoadjuvant mode.

\section{LITERATURE}

1. Nerodo G. A. Relapses of vulva cancer // Proceedings of the $\mathrm{Vl}$ all-Russian Congress of oncologists, 2005, p. 382-383.

2. Cherenkov V. G., I. V. Alexandrov, E. S. Egorov. A rare case of vulva cancer in a young woman.// Tumors of the female reproductive system (mammology/oncogynecology), № 1-2, 2013, p. 24.

3. Cherenkov V. G., Sannikova M. V. Screening and treatment of risk groups and external genital cancer. LAP LAMBERT Academic Publishing, 2018, 58p.

4. Zharov, A. V., Vazhenin A. V. Optimization of treatment of patients with vulva cancer. Chelyabinsk, 2005, 131c.

5. Ate van der Zee. Modern treatment of vulva cancer. Topical issues of oncogynecology//Practical Oncology, vol. 10, № 2, 2009, p. 84-85.

6. Krikunova L. I., Kaplan M. A., Rykova E. V. the Role of photodynamic therapy in the treatment of vulva cancer// 1 international conference. Obninsk, 1999.p. 32-33.

7. Nerodo G. A. Complex treatment of patients with recurrence of vulvar cancer/ Scientific-practical conference with international participation "Improvement of medical care for cancer, including topical problems of pediatric Hematology and Oncology. The national cancer program."YII Congress of oncologists of Russia, vol. 2, Moscow, 2009, pp. 61-62.8.

8. Urmancheyeva A. F. Epidemiology of cancer of the vulva. Risk and prognosis factors. Practical Oncology, vol. 7, № 4, 189-196, 2006.

9. Knapstein P. G. Erweiterte Behandlungsmöglichkeiten Vulvakarzinomas durch das Verfahren plastic reconstructive P. G. Knapstein, M. Mahlke, W. Poleska, W. Zeuner II Zbl.Gynekol.-1985.Bd.107, N 24.-p.1479-1487.
10. Patent No. 2580665 for invention dated $11.11 .14 \mathrm{~g}$ vniigpe. V. G. Cherenkov A. B., Egorova E. S., Shpenkov, A. A., Aleksandrova, M. S. Pilosov. 